\title{
ANALYTICAL VISCOUS FLOW MODEL AND TEST VALIDATION OF A SWIRL INJECTOR FOR ROCKET ENGINE APPLICATION
}

\author{
G. Fiore ${ }^{1}$, C. Bach ${ }^{2}$, J. Sieder ${ }^{2}$, and M. Tajmar ${ }^{2}$ \\ ${ }^{1}$ Università di Pisa \\ 8 Via Caruso, Pisa 56122, Italy \\ ${ }^{2}$ Technische Universität Dresden \\ 32 Marschnerstrasse, Dresden 01307, Germany
}

\begin{abstract}
The generally adopted flow model inside a swirl injector, widely used injection concept for propulsive applications, relies upon the hypothesis of ideal flow neglecting the fluid viscosity effects. This model showed significant prediction errors with relatively high viscosity propellants, often leading to the need of an experimental characterization of the injection elements. In this paper, an analytical approach is presented, which includes the effects of viscous diffusion on the injector performance leading to a close form flow solution. The built model is thus experimentally validated testing a liquid oxygen (LOx) and an ethanol injector: the good agreement between the model and the experimental results leads to the construction of the injectors operational maps describing the injector behavior even in the presence of viscous effects.
\end{abstract}

\section{NOMENCLATURE}

$A_{\text {film }} \quad$ Fluid film cross-sectional area

$A_{n} \quad$ Injector nozzle cross-sectional area

$C \quad$ Dimensionless circulation

$d S \quad$ Surface element

$h_{\text {sw }} \quad$ Swirling film thickness

$L \quad$ Characteristic flow length

$l_{\mathrm{sw}} \quad$ Swirl chamber wetted path length

$\dot{m} \quad$ Mass flowrate

$\dot{m}_{\text {defect }}$ Viscous flowrate defect

$\dot{m}_{\text {ideal }}$ Ideal flowrate

$n \quad$ Number of tangential inlets

$p_{c} \quad$ Combustion chamber pressure

(C) The Authors, published by EDP Sciences. This is an open access article distributed under the terms of the Creative Commons Attribution License 4.0 (http://creativecommons.org/licenses/by/4.0/). 
$p_{\text {feed }} \quad$ Injector feeding pressure

$\mathfrak{R} \quad$ Trajectory radius of curvature

Re Reynolds number

$\mathrm{Re}_{\mathrm{sw}}$ Swirl chamber Reynolds number

$\operatorname{Re}_{x} \quad$ Streamwise coordinate Reynolds number

$r_{\mathrm{gc}} \quad$ Gaseous core radius

$r_{\text {in }} \quad$ Tangential inlets radius

$R_{\text {in }} \quad$ Chamber swirl arm

$R_{N} \quad$ Injector nozzle radius

$R_{\mathrm{sw}} \quad$ Swirl chamber radius

$U \quad$ Characteristic flow velocity

$u_{0} \quad$ Unperturbed velocity profile

$u_{(y)} \quad$ Stratified velocity profile

$v \quad$ Azimuthal velocity

$V \quad$ Total velocity

$V_{N} \quad$ Total velocity in the injector nozzle

$x_{\mathrm{sw}} \quad$ Swirl path coordinate

$z_{N} \quad$ Injector axial length

$\beta \quad$ Flow spread angle

$\Delta p \quad$ Injector pressure drop

$\delta^{*} \quad$ Displacement thickness

$\varphi \quad$ Passage fullness coefficient

$\mu \quad$ Massflow coefficient

$\mu_{l} \quad$ Fluid viscosity

$\mu_{\text {vis }} \quad$ Viscous massflow coefficient

$\rho \quad$ Fluid density

$\vartheta \quad$ Azimuthal coordinate

\section{INTRODUCTION}

The use of a swirl injector to feed the combustion chamber of a rocket engine has proven to be a powerful choice in terms of atomization and mixing efficiencies [1], combustion stability margin, and throttleability [2] together with high massflow per element. These features are gained through the tangential introduction of the propellant in the swirl chamber that realizes an angular momentum-driven flow development.

Nevertheless, the high fluid/wall interfacial areas make this injection concept very sensitive to viscosity effects [2] since the Boundary Layer (BL) height can become comparable to the dimensions of the spreading propellant film thickness.

The existing flow model, developed in the late 1970s by Bazarov et al. [1], relies upon the hypothesis of inviscid flow: solving the flow equation in this frame gives, in fact, the possibility to correlate the applied pressure drop $\Delta p$ 


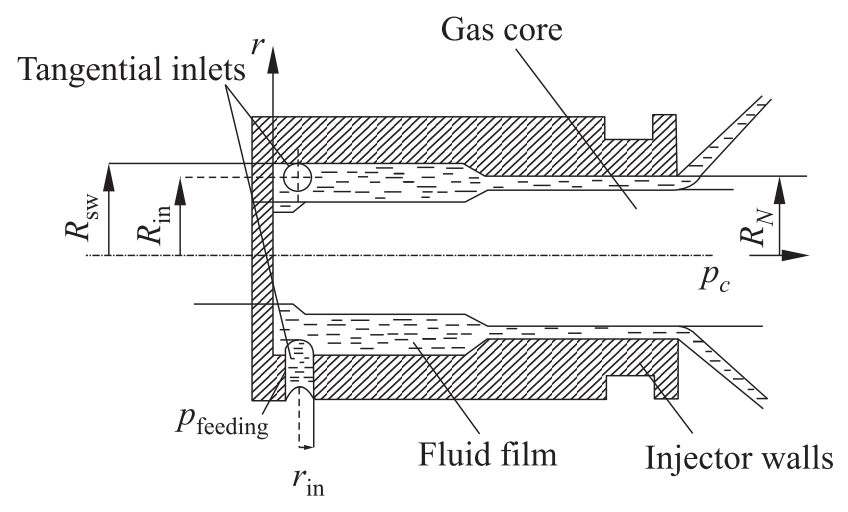

Figure 1 Swirl injector scheme [1] (modified)

to the expelled massflow $\dot{m}$ by means of a dimensionless coefficient $\mu$ known as massflow discharge coefficient:

$$
\dot{m}=\mu \pi R_{N}^{2} \sqrt{2 \rho \Delta p}
$$

where $R_{N}$ is the radius of the injector outlet as shown in Fig. 1 and $\rho$ is the density of the driven propellant. Defining two other dimensionless groups, the dimensionless circulation $C$ and the film passage fullness coefficient $\varphi$ as

$$
C=\frac{R_{N} R_{\mathrm{in}}}{n r_{\mathrm{in}}^{2}} ; \quad \varphi=\frac{A_{\mathrm{film}}}{A_{N}}=1-\left(\frac{r_{\mathrm{gc}}}{R_{N}}\right)^{2},
$$

the Bazarov inviscid theory is able to correlate the massflow coefficient $\mu$ to the injector geometrical features:

$$
\mu=f\left(R_{N}, R_{\text {in }}, r_{\text {in }}, n\right)
$$

where $R_{N}, R_{\text {in }}$, and $r_{\text {in }}$ are the radii of the nozzle outlet, the injection arm, and the tangential inlets, respectively, geometrical dimensions of the injector as shown in Fig. $1 ; n$ is the number of tangential inlets; $A_{\text {film }}$ and $A_{N}$ are the crosssectional areas of the fluid film and the nozzle outlet; and $r_{\mathrm{gc}}$ is the gaseous core radius. Experimental data for this injection concept have been obtained within the SMART Rockets project [3], in which a 500-newton liquid propellant engine is designed with the goal of propel a payload-carrying sounding rocket [4]. This engine in fact features a coaxial swirl injector assembly in which LOx and ethanol are driven to feed an oxide ceramic matrix composite (OCMC) combustion chamber. Experimental data obtained with LOx and ethanol, two fluids with relatively low and high viscosities (Table 1), confirm the good prediction 
Table 1 Propellants viscosities $[5,6]$

\begin{tabular}{lcc}
\hline \multirow{2}{*}{ Propellant } & \multicolumn{2}{c}{$\mu_{l}, \mathrm{mPa} \cdot \mathrm{s}$} \\
\cline { 2 - 3 } & $10 \mathrm{bar}$ & $20 \mathrm{bar}$ \\
\hline $\mathrm{LOx}_{\text {sat }}$ & 0.097 & 0.082 \\
$\mathrm{LN}_{2 \text { sat }}$ & 0.075 & 0.05 \\
Ethanol & 1.17 & 1.25 \\
\hline
\end{tabular}

accuracy of the Bazarov model in the low viscosity case while significant deviations are observed in the second case [7] (see section 4). This prompts viscous diffusion effects to be the cause of the mismatch between the ideal theory and the experiments and at the same time highlights the need for a model accounting for the fluid viscosity to characterize the injector with a pressure-massflow correlation.

In this paper, an analytical approach to account for viscosity effects on the injector discharge capability is presented: the BL structure inside the swirl chamber is modeled in terms of the ideal flow parameters obtaining a close form solution for the viscous discharge coefficient. The obtained results avail to build the operative map of the swirl injector that is then compared to experimental data obtained via cold flow testing of the injectors.

\section{VISCOUS FLOW MODEL}

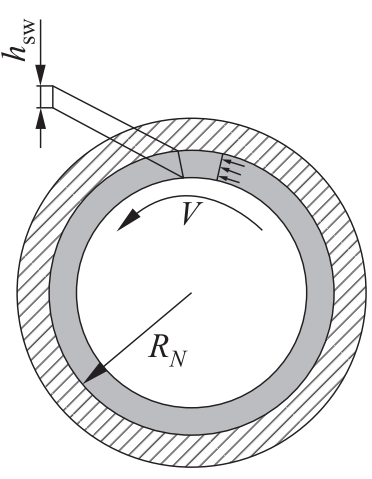

Figure 2 Flow configuration
The first step for the inclusion of viscous effects into the flow model is to give a proper definition of the Reynolds number for the flow. This dimensionless parameter, in fact, displays the relative importance of inertial and viscous effects acting in the problem:

$$
\operatorname{Re}=\frac{\text { Inertial Force }}{\text { Viscous Force }}=\frac{\rho U L}{\mu_{l}},
$$

being $U$ and $L$ the characteristic velocity and length of the flow and $\mu_{l}$ the viscosity of the fluid. In order for this parameter to be representative of the flow viscous behavior, appropriate values should be chosen for the characteristic

length and velocity. With reference to Fig. 2, by definition of Reynolds number, what matters is the length across which the fluid is pushed by the inertial forces. In this particular flow configuration, this length is well represented by the film thickness $h_{\mathrm{sw}}$ while, due to the three-dimensional (3D) nature of the problem, the reference velocity should be taken as the total velocity $V$.

Specializing the thickness and the total velocity at the injector nozzle exit section, so to earn ease of representation, and rearranging the Reynolds definition with the help of the ideal flow relations [1], the total velocity can be expressed in 
terms of the injector massflow. In this way, the overall Reynolds number inside the swirl chamber can be defined as a function of the injector mass flowrate:

$$
\operatorname{Re}_{\mathrm{sw}}=\frac{\rho V_{N} h_{\mathrm{sw}}}{\mu_{l}}=\frac{\varphi \dot{m}}{2 \pi \mu R_{N} \mu_{l}}
$$

where the thickness of the annular section has been expressed using the thin annulus approximation valid for $h_{\text {sw }} \ll R_{N}$ (if this hypothesis does not hold, the actual thickness is computed without simplifications) and the definition of passage fullness coefficient $\varphi$ :

$$
A_{\mathrm{film}}=\varphi \pi R_{N}^{2}=\pi R_{N}^{2}-\pi\left(R_{N}-h_{\mathrm{sw}}\right)^{2} \approx 2 \pi R_{N} h_{\mathrm{sw}} .
$$

Hence,

$$
h_{\mathrm{sw}}=\frac{\varphi R_{N}}{2} .
$$

As an example, Table 2 shows the values of the defined swirl Reynolds number for different flows of liquid nitrogen $\left(\mathrm{LN}_{2}\right), \mathrm{LOx}$, and ethanol, specified for the LOx and ethanol injectors built within the SMART Rockets project [4].

\begin{tabular}{|c|c|c|}
\hline \multirow{2}{*}{ Propellant } & \multicolumn{2}{|c|}{$\operatorname{Re}_{\mathrm{sw}}$ at $125 \mathrm{~g} / \mathrm{s}$} \\
\hline & 10 bar & 20 bar \\
\hline $\mathrm{LOx}$ & 197200 & 232000 \\
\hline $\mathrm{LN}_{2}$ & 255000 & 382600 \\
\hline Ethanol & 12600 & 12000 \\
\hline
\end{tabular}

The values of the Reynolds number con-

Table 2 Swirl Reynolds numbers

firm what has been observed in the injectors

tests: for $\mathrm{LOx}$ and $\mathrm{LN}_{2}$, the Reynolds number is one order of magnitude higher than the one of the ethanol, no sensible viscosity effects should be noticed so that the inviscid model should be capable of accurately predicting the empirical results. The opposite happens on the ethanol line since the very low Reynolds, justified by higher viscosity of the fluid, makes the problem difficult to be realistically described when neglecting viscosity effects that play an important role in the flow development.

What happens is that viscous diffusion causes a massflow reduction through the injector because of the velocity stratification near the wall. The nonslip condition at the wall forces the particles entrained in the flow near the swirl chamber wall to have zero velocity but moving away from the injector surface, the flow gains its nominal velocity since viscosity effects are confined in the vicinity of the wall. This causes a stratification of the velocity profile that can no longer be considered uniform but radially varying. Recalling the concept of the BL theory, it can be stated that being the BL a region near the solid wall where all the viscosity effects are confined, the velocity varies from zero at the wall to the inviscid value at the BL edge since outside the BL, no viscosity effect is felt; thus, the fluid behaves as it would in an inviscid flow (Fig. 3).

The consequence of the viscosity-induced velocity profile stratification is that in the viscous case, the massflow entrained in the BL thickness is actually lower 


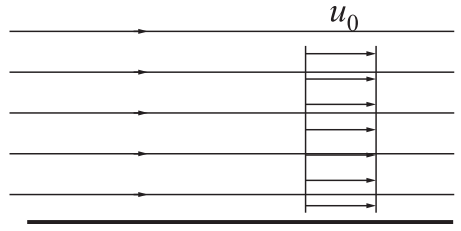

(a)

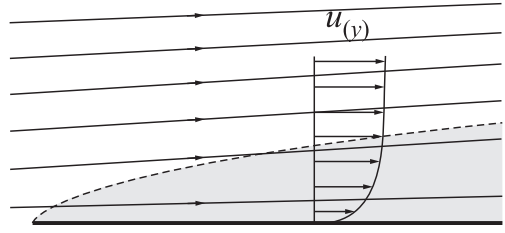

(b)

Figure 3 Boundary layer theory: ideal $(a)$ and viscous $(b)$ velocity profiles

than the one that would be entrained in the same length in an inviscid flow. Since the velocity outside the BL is uniform and equal in both cases, it can be stated and verified by direct integration that the overall massflow in the viscous case is reduced because of viscous diffusion effects:

$$
\int_{\text {viscous }}^{h} \rho \vec{V}(r) \overrightarrow{d S}<\int_{\text {inviscid }}^{h} \rho \vec{V} \overrightarrow{d S} .
$$

The curious fact is that this process of massflow reduction takes places in both ethanol and LOx flows with the same mechanism: however, being the BL height inversely proportional to the square root of the Reynolds number, in the LOx flow, Re is so high that viscosity effects are confined in an extremely thin region near the wall, so that the decrease in massflow becomes negligibly small. This rather simple explanation of the viscous diffusion in the injector flow well describes what has been observed experimentally for the two different flows.

The focus is, therefore, shifted in finding the velocity distribution in the BL and use (4) to obtain the massflow defect. In particular, what matters is the BL profile at the end of the injector duct: the BL thickness increases along the axial coordinate of the injector and the representative figure for the mass defect is the integration of the velocity profile at the injector outlet where the viscosity-dominated region has the highest thickness. A convenient choice that suites this problem could be the use of the definition of the BL displacement thickness $\delta^{*}$. This characteristic length value is the thickness that should be added to the wall (or equivalently deprived to the flow near the wall) in order to obtain an equivalent uniform flow, with uniform velocity equal to that outside the BL, whose massflow equals the one that would be obtained by integration of the actual velocity profile, as sketched in Fig. 4.

The next step would be to evaluate the displacement thickness at the injector outlet and then treat the flow inside the injector as uniform like in the inviscid case, but on a fictitious geometry in which the wall is shifted by a $\delta^{*}$ length. In this way, the massflow flowing through this fictitious geometry is the actual massflow through the injector including viscous mass defects. 


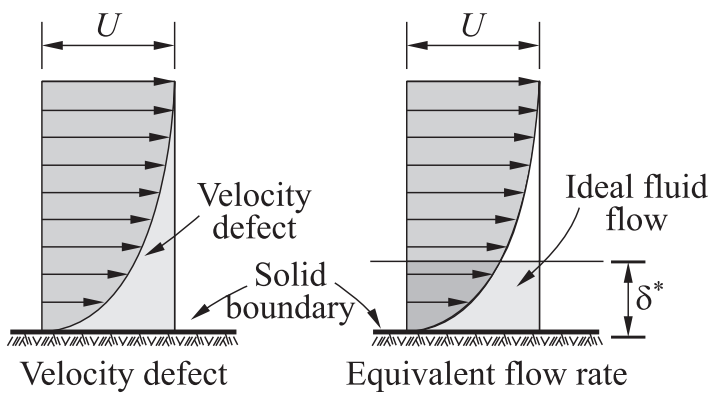

Figure 4 Boundary layer displacement thickness [8]

Evaluation of the BL mattering thicknesses is, however, not a trivial challenge, especially due to the $3 \mathrm{D}$ nature of the flow configuration. Anyway, known solutions for pretty different flow configurations can be taken as a reference. The relevant differences in terms of geometric and fluid-dynamic effects between the actual and the reference flow configurations can thus be discussed to assess the limits of validity and the differences that those effects bring on the growth of the BL thickness that is the primary quantity of interest. The Blasius solution for the BL developing across a flat plate flow can be taken as a reference: in this simple flow configuration, no pressure gradients are experienced so that the BL grows naturally with the flow coordinate. The solution for the growth of the displacement thickness as a function of the flow coordinate is [9]:

$$
\delta^{*}(x)=1.7208 \frac{x}{\sqrt{\operatorname{Re}_{x}}}=1.7208 \sqrt{\frac{\mu_{l} x}{\rho V}}
$$

where the $x$ coordinate represents the portion of the wall length wett by the fluid. The different factors influencing the growth of the BL in the actual configuration with regard to the reference one come from two effects:

(1) presence of a negative axial pressure gradient toward the injector nozzle due to propellant acceleration; and

(2) effect of curvature developing centrifugal forces inside the flow.

The first effect is usually beneficial in terms of growth of the BL since negative pressure gradients along the streamwise direction are stabilizing and retard the BL's thickness growth. The second effect requires a little bit more attention since centrifugal forces set up due to the convex curvature build up a radial pressure stratification, according to the local equilibrium of a flow element, the pressure differential in the radial direction is:

$$
(d p)_{r}=\rho \frac{V^{2}}{\mathfrak{R}} d r
$$


where $\mathfrak{R}$ is the local radius of curvature of the flow, of the order of the chamber radius, and depending on the axial-to-azimuthal velocity ratio. The important fact is that the pressure gradient in the radial direction is positive, meaning that the flow is pushed against the wall by centrifugal effects (centrifugal pumping), leading to a further decrease of the BL thicknesses with respect to the reference ones.

As a result, inclusion of the effects of the different flow configurations with regard to the Blasius one gives that the BL's properties, in particular, the displacement thickness, grow in a slower fashion with the flow coordinate meaning that the use of the Blasius profile turns up in a conservative overestimation of the viscosity effects. This fact is really encouraging because if it were the opposite, namely, the BL thickness was underestimated, the Blasius model could no longer have been used because the faster growth of the BL in the injector would have probably lead to separation of the flow, condition at which the BL behaves in a totally different fashion with respect to the reference one.

Drawing conclusions, the use of a Blasius profile still well describes the mechanisms that lead to the massflow defect bringing an acceptable overestimation of the BL thicknesses with the advantage of a handy analytical solution amenable to be easily accommodated into the swirl injector flow model. Accordingly, the BL's displacement thickness for the swirl injector can be expressed in a convenient way using the definition for $\operatorname{Re}_{\mathrm{sw}}(2)$ and the film thickness (3):

$$
\delta^{*}\left(x_{\mathrm{sw}}\right)=1.7208 \sqrt{\frac{\mu_{l} x_{\mathrm{sw}}}{\rho V_{N}} \frac{h_{\mathrm{sw}}}{h_{\mathrm{sw}}}}=1.7208 \sqrt{\frac{\varphi R_{N} x_{\mathrm{sw}}}{2 \mathrm{Re}_{\mathrm{sw}}}}
$$

where the coordinate $x_{\mathrm{sw}}$ is now the curvilinear abscissa of the helical path of the flow through the swirl chamber, representing the length of the wet wall's surface. This is the mattering length for the evaluation of the BL growth along the path of the fluid inside the injector as sketched in Fig. 5.

This parameter can be found by geometrical considerations about the helicallike pattern of the flow as it is intuitive the shorter the pitch of the helix, the longer the wet surface and vice versa, since the axial length of the helix is fixed by the injector size. An exact characterization of the swirled path can be done with the help of the inviscid theory using the result for the spread angle or equivalently the axial-to-total velocity ratio. The use of the inviscid theory for the evaluation of the path turns to be helpful in obtaining a "linearized" viscous result as a correction of the inviscid one that stands as a reference.

In a cylindrical reference frame, the parametric equations of a helical curve of radius $R$, pitch $p$, and azimuth $\vartheta$ are:

$$
x=R \cos \vartheta ; \quad y=R \sin \vartheta ; \quad z=\frac{p}{2 \pi} \vartheta .
$$




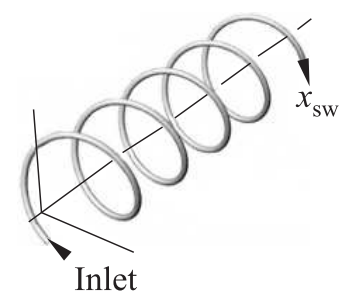

Figure 5 Helical path of the swirling flow inside the injector

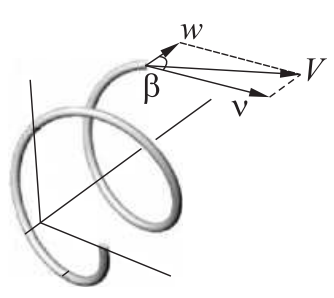

Figure 6 Velocity ratio and spread angle

The spread angle $\beta$ of the helix can be related to the inviscid flow parameters via

$$
\cos \beta=\frac{w}{V}=\frac{\mu}{\varphi}
$$

and by geometrical analysis, the relation holding between the pitch and the spread angle (Fig. 6) is:

$$
\tan \beta=\frac{2 \pi R}{p} .
$$

The goal of the geometrical analysis is to find the variation of the flow coordinate $x_{\mathrm{sw}}$ and, in particular, its value at the end of the nozzle $\left(z=z_{N}\right)$ as a function of the flow parameters. This length can be found by straight integration of the helix equation; with few calculus, it is obtained:

$$
l_{\mathrm{sw}}=\int_{0}^{x_{\mathrm{sw}}\left(z_{N}\right)} d s
$$

with

$$
d s=\sqrt{d x^{2}+d y^{2}+d z^{2}}=d z \sqrt{\left(\frac{2 \pi R}{p}\right)^{2}+1}=d z \sqrt{\tan ^{2} \beta+1}=\frac{d z}{\cos \beta}
$$

and with the variable substitution the wet path length $l_{\mathrm{sw}}=x_{\mathrm{sw}}\left(z_{n}\right)$ is obtained:

$$
l_{\mathrm{sw}}=\int_{0}^{z_{N}} \frac{d z}{\cos \beta}=\frac{\varphi}{\mu} z_{N} .
$$

Hence, it is now possible to express the displacement thickness evaluated at the nozzle outlet section. Leading parameter for the expression of the viscous mass defect:

$$
\delta^{*}\left(z_{N}\right)=1.7208 \sqrt{\frac{\varphi R_{N} l_{\mathrm{sw}}}{2 \mathrm{Re}_{\mathrm{sw}}}} .
$$


Being able to pass into the equivalent uniform flow in which the wall thickness is shifted by a displacement thickness length and remaining in the thin film approximation, the defect massflow can be found via a simple proportion. In fact, dealing with uniform flows, the defect massflow and the displacement thickness stand in the same relation of the ideal massflow and the total thickness film, the same can be stated for the massflow coefficients:

$$
\frac{\dot{m}_{\text {defect }}}{\delta^{*}}=\frac{\dot{m}_{\text {ideal }}}{h_{\mathrm{sw}}} \Rightarrow \dot{m}_{\text {defect }}=\frac{\delta^{*}}{h_{\mathrm{sw}}} \dot{m}_{\text {ideal }} .
$$

Hence, with the definition (3) of the film thickness,

$$
\dot{m}_{\mathrm{vis}}=\dot{m}_{\text {ideal }}-\dot{m}_{\text {defect }}=\dot{m}_{\text {ideal }}\left(1-\frac{2 \delta^{*}}{\varphi R_{N}}\right) \text {. }
$$

Finally, using (5), the relation for the viscosity-corrected massflow discharge coefficient writes:

$$
\mu_{\mathrm{vis}}=\mu\left(1-1.7208 \sqrt{\frac{2 l_{\mathrm{sw}}}{\varphi R_{N} \mathrm{Re}_{\mathrm{sw}}}}\right) .
$$

This relation finally gives the massflow coefficient of the injector accounting for viscous losses. The new dimensionless parameter $\mu_{\mathrm{vis}}$ has the same task of the ideal one for performance prediction: relate the pressure drop to the massflow established in the injector via (1). This time, the massflow coefficient does not only depend on the injector's basic geometry like it was in the ideal model.

The longitudinal dimension of the injector is considered since it fixes the length of the wet surface on which viscous effects act. There is also a massflow dependence through the swirl Reynolds number: the higher the velocity of the flowing fluid, the slower the growth of the BL, meaning that going towards higher massflows (increasing Re) the viscosity effects are less and less important. Substitution of the $\operatorname{Re}_{\mathrm{sw}}$ definition (2) into (6) allows to plot the $\mu_{\mathrm{vis}} / \dot{m}$ characteristic curve and to compare it to the inviscid theory.

Figure 7 shows the trend of the massflow coefficients of the LOx and ethanol injectors compared to the ideal theory.

As Fig. 7 shows, there is no sensible shift between ideal and viscous model on the LOx injector, the low viscosity and, in particular, the high Reynolds number realized in the injector make the ideal model an accurate tool for the injector performance prediction. A different situation is depicted on the ethanol injector, ideal and viscous models give conflicting results leading to an evident shift in the massflow coefficient prediction. Even the trend to increase the discharge capability toward high massflows is more pronounced emphasizing the ethanol injector sensitivity to Reynolds number changes. 

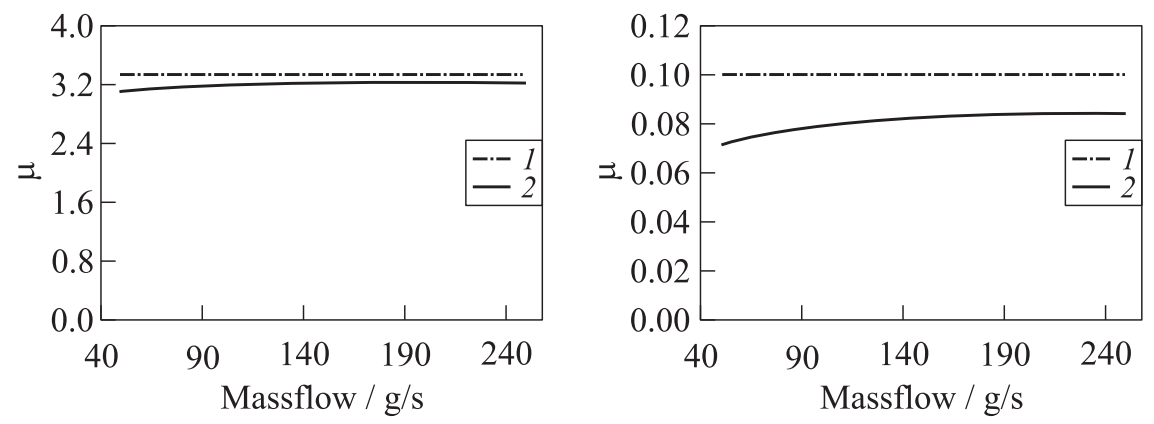

Figure 7 Liquid oxygen (a) and ethanol (b) ideal (1) and viscous (2) discharge coefficients

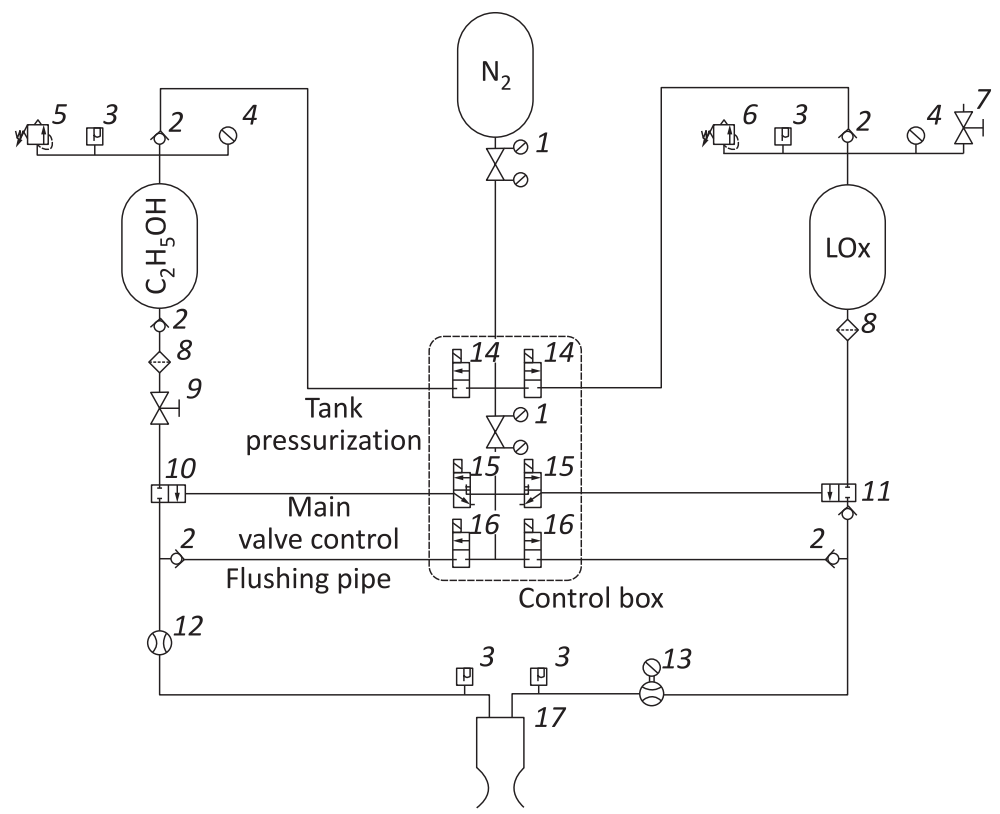

Figure 8 Test bench scheme [10]: 1 - pressure regulator; 2 - back-pressure valve; 3 - pressure sensor; 4 - manometer; 5 - pressure control valve with manual pressure release; 6 - pressure control valve; 7 - venting valve; 8 - filter; 9 - gate valve; 10 - main valve ethanol; 11 - main valve LOx; 12 - flowmeter; 13 - venturi tube with differential pressure manometer; 14 - solenoid control valve $(2 / 2$-way); 15 solenoid control valve (3/2-way); 16 - solenoid control valve (2/2-way); and 17 combustion chamber 


\section{EXPERIMENTAL SETUP}

To validate the built viscous model, the experimental apparatus realized within the SMART Rockets project [10] has been set for the cold flow testing of the injectors. A scheme of the test bench is shown in Fig. 8 .

The test facility built for the sake of testing a 500-newton LOx-ethanol propelled engine features two propellant tanks for the LOx (cryogenic proven) and ethanol, respectively, both pressurized by a gaseous nitrogen tank and connected with the injectors. Several sensors are installed on the propellants lines: tempera-

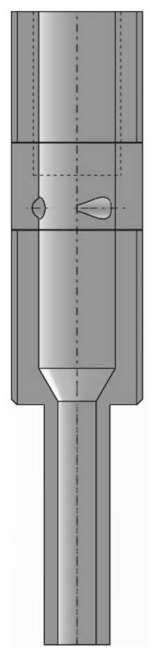

(a)

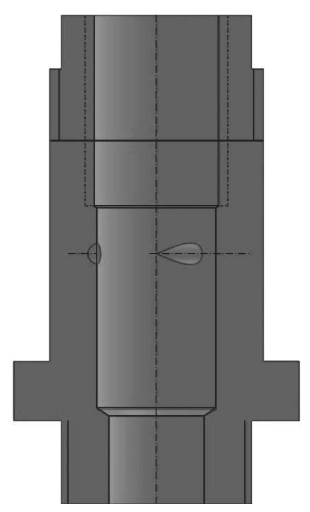

(b)

Figure 9 The CAD section view of the LOx $(a)$ and ethanol $(b)$ injectors [7] ture sensors are mounted in the $\mathrm{LOx}$ tank to control the vapor fraction and to monitor the fueling cycles of the cryogenics, pressure sensors are mounted inside the tank and before the injectors to control the line pressures. Massflow sensors on each line measure the flowrates and, in particular, the LOx massflow sensor is also able to measure the fluid density accounting for vapor presence in the cryogenic that always works near saturation conditions.

The connection between the test bench and the injector has been realized using a copper pipe on the ethanol line and a thermally insulated pipe on the LOx line, insulation of the LOx line and cooling cycles proved in fact to be a critical challenge for obtaining vapor free shots of the cryogenic during the test campaigns. Figure 9 shows the CAD (computer-aided design) section views of the two injectors used for the tests.

Both injectors are fed via three tangential inlets connected to the feeding lines of the test bench and are designed to work in a coaxial fashion. Both injectors have been previously designed using the inviscid theory $[3,10]$ and this gave a substantial shift between predictions and test observations. It is through the validation of the viscous flow model that a more reliable prediction of the injectors massflow coefficients will be obtained, giving the possibility to readjust the injectors internal geometries and to adapt the coaxial assembly into the propulsion system.

The manufacturer of the pressure sensors claims a measurement error of 0.2 bar while the maximum massflow error is $5 \mathrm{~g} / \mathrm{s}$, satisfactory values since the 
tests are realized in the neighborhood of 10-bar pressure and $150 \mathrm{~g} / \mathrm{s}$ massflow, way far from the sensor error range. Attention should be paid to realizing stable and steady flows since both results of ideal and viscous theory were obtained under the hypothesis of steady flow field.

\section{TEST RESULTS}

The test campaign has been conducted using ethanol and the simulant fluid $\mathrm{LN}_{2}$ in place of $\mathrm{LOx}$ for safety reasons. Being $\mathrm{LN}_{2}$ a cryogenic fluid with similar transport properties of $\mathrm{LOx}$, the results can be reliably scaled. The injectors have been tested in two different cold flow fashions, single flow and combined ethanol- $\mathrm{LN}_{2}$ flow in order to check if any mutual throttling effect exists when the two injectors work in the coaxial fashion, providing a mixing zone to the fluids before the jet expulsion. Arranged the test bench for the particular test to be run, several shots were obtained with the goal of reaching steady-state conditions for a representative time interval. The cryogenic shots were always preceded by cooling preshots in order to cool down the LOx line obtaining shorter settling time for the fluid to become vapor-free, realizing thus steadier test runs.

For both the prescribed flow fashions, a pressure range 5-20 bar has been covered with a corresponding massflow range 50-200 g/s in order to conveniently simulate the range of interest for the rocket engine the injectors belong to. Steady-state values of pressures, massflows, and densities are evaluated and compared to the ideal and viscous predictions and Eq. (1) is used with the measured data to obtain the observed values of the massflow coefficients.

The first result obtained is that no sensible throttling effect has been observed with the injectors working in combined flow fashion. This is an important result since the combined flow fashion is the actual working condition of the injectors in the engine. The absence of mutual throttling effects means that in the design phase, the operative map of each injector can be used by itself in order to set the geometric and pressure parameters, then the injectors can be assembled maintaining the same pressure-flowrate relations.

Results in terms of massflow coefficient are shown in Figs. 10 and 11 [7]; for the LOx injector, previous results obtained with LOx cold flow tests are also displayed:

- the LOx injector results show a good agreement between empirical observation and theory, once again there is no substantial difference between ideal and viscous theory given the high values of the Reynolds number for this kind of flow. A certain degree of scattering of the test results is observed due to the density fluctuations of the cryogenics, symptom of a certain vapor fraction in the fluid also confirmed by the slightly lower density observed, especially in the lower massflow range, index of lower 


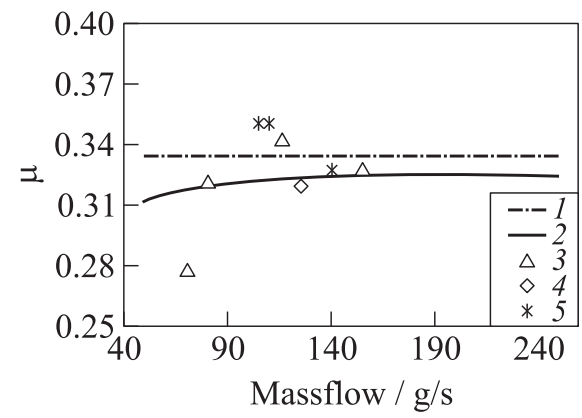

Figure 10 The LOx injector test results: 1 - ideal; 2 - viscous; 3 - $\mathrm{LN}_{2}$-tests; $4-\mathrm{LN}_{2}$-combined; and $5-$ LOx-tests

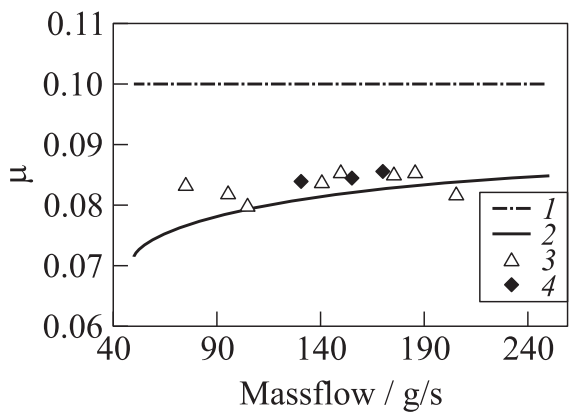

Figure 11 Ethanol injector test results: 1 - ideal; 2 - viscous; 3 - Ethanoltests; and 4 - Ethanol-combined

injection pressure thus reduced saturation temperature of the cryogenic; and

- results obtained with the ethanol injector confirm with excellent accuracy the viscous theory: in addition to being very close to the predicted values, the test results confirm the trend of discharge capability reduction towards decreasing flowrates indexes of lower Reynolds numbers thus higher influence of viscous effects in the flow. In addition, the analytical prediction slightly underestimates the discharge coefficient (overestimates the viscous effects) and this is in accordance with the hypotheses holding behind the viscous model, in particular, the use of a Blasius BL profile parametrized around the swirl path. This assumption in fact neglects the beneficial pres-

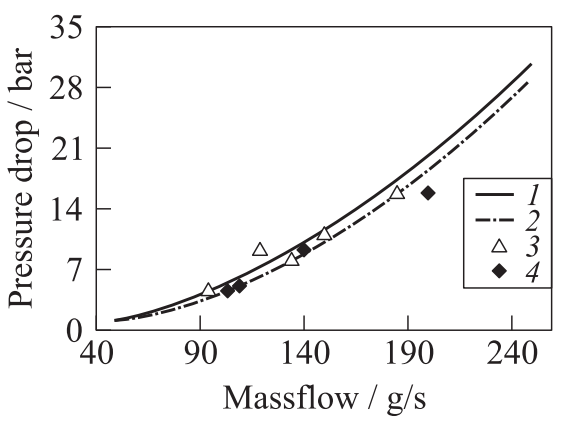

Figure 12 The LOx injector map: 1 viscous pressure drop; 2 - inviscid pressure drop; 3 - test LOx; and 4 - test $\mathrm{LN}_{2}$

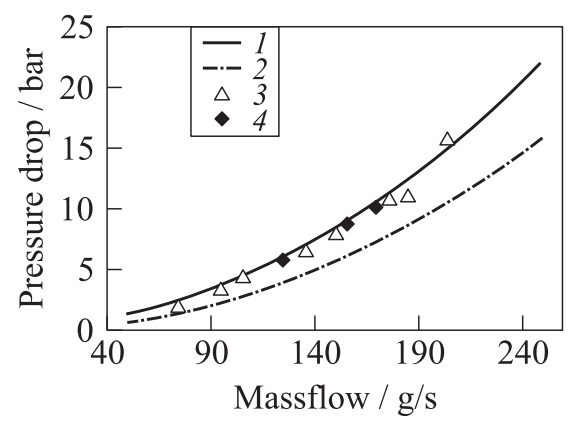

Figure 13 Ethanol injector map: 1 viscous pressure drop; 2 - inviscid pressure drop; 3 - test single; and 4 - test combined 
sure gradients coming from the fluid centrifugation that retard the growth of the BL slightly increasing the discharge capability of the injector.

Using the new definition of the discharge coefficient (6), it has been possible to build the new pressure-massflow correlation and to design the operational maps of the injectors. Figures 12 and 13 show the operative maps of both injectors together with the obtained test results highlighting the difference between ideal and viscous maps [7].

\section{CONCLUDING REMARKS}

A viscous flow model has been built for the swirl injector, obtaining an analytical expression for the massflow discharge coefficient, depending both on the injector geometrical features and on the fluid's transport properties that characterize the flow BL structure. A cold flow test campaign on the injector has been conducted and confirmed the predictions of the viscous flow model: test results confirmed with excellent accuracy the viscous predictions also highlighting the influence of the Reynolds regime on the injector discharge capability. This is observed very clearly in the ethanol injector since no substantial difference between ideal and viscous flow model holds for the low viscosity case of the LOx injector. Through the definition of the viscous discharge coefficient, the injectors have been characterized via pressure-massflow correlations, key aspect for the design phase of the injector themselves and for the assignment of the line pressures in the propulsion assembly, otherwise done in an experimental way to account for the inappropriateness of the ideal model to describe highly viscous flows. The presented model represents thus a powerful tool for the understanding and design of this injector concept even when propellant viscosity effects cannot be neglected.

\section{ACKNOWLEDGMENTS}

The authors would like to thank Dr. Olaf Przybilski, team leader of the project, and all the "SMART Rockets" student team of the "TU Dresden" for their support during the experimental phase; Ing. Maximilian Nürnmberger, responsible for the test bench control; Prof. Luca D'Agostino of "Università di Pisa" and Ing. Lorenzo Vallini for their wise suggestions during the paper writing phase.

\section{REFERENCES}

1. Bazarov, V., V. Yang, and V. Puri. 2004. Design and dynamics of jet and swirl injectors. Liquid rocket thrust chambers: Aspects of modeling, analysis, and design. 
Eds. M. Popp, J. Hulka, V. Yang, and M. Habiballah. Progress in astronautics and aeronautics ser. Reston, VA: AIAA. 200:19-104.

2. Zong, N., and V. Yang. 2008. Cryogenic fluid dynamics of pressure swirl injectors at supercritical conditions. Phys. Fluids 20(5):056103.

3. Bach, C., J. Sieder, and O. Przybilski. 2014. STERN Project documentation. TU Dresden.

4. Bach, C., J. Sieder, M. Nürnmberger, N. Voigt, O. Przybliski, and M. Tajmar. 2014. Proceedings of the SMART Rockets project: Design development and first measurement results of a $500 \mathrm{~N}$ LOx/ethanol combustion chamber. Deutscher Luftund Raumfahrtkongress 2014.

5. Hanley, M., R. D. McCarty, and W.M. Haynes. 1974. The viscosity and thermal conductivity coefficients for dense gaseous and liquid argon, krypton, xenon, nitrogen and oxygen. Cryogenic Division, National Bureau of Standards.

6. Tanaka, Y., Y. Matsuda, H. Fujiwara, et al. 1987. Viscosity of (water + alcohol) mixtures under high pressure. Int. J. Thermophys. 8(2):147-163.

7. Fiore, G. 2015. Design of the flight version combustion chamber head and coaxial swirl injector for a LOx-ethanol rocket engine. Università di Pisa / TU Dresden. STERN Project documentation.

8. Goyal, R. 2013. Boundary layer equations. Malaviya National Institute of Technology.

9. Blasius, H. 1908. Grenzschichten in Flüssigkeiten mit kleiner Reibung. Z. Math. Phys. 56:1-37.

10. Bach, C., J. Sieder, O. Przybliski, and M. Tajmar. 2013. Smart Rockets: development of a $500 \mathrm{~N} \mathrm{LOx} /$ ethanol-sounding rocket for the DLR STERN programme. Deutscher Luft-und Raumfahrtkongress.

11. Yang, V., M. Habiballah, J. Hulka, and M. Popp, eds. 2004. Liquid rockets thrust chambers: Aspects of modeling, analysis, and design. Progress in astronautics and aeronautics ser. Reston, VA: AIAA. Vol. 200. 500 p.

12. Eberhart, C., D. Lineberry, and M. Moser. 2010. Effects of variable chamber pressure on a swirl coaxial injector: A cold flow study. AIAA Paper No. 2010-6665. 